\title{
Handelsbeziehungen Schweiz - EU im globalen Kontext
}

\author{
Charlotte Sieber-Gasser *
}

Dass das globale, regelgebundene Handelssystem gegenwärtig unter Druck steht, wirkt sich auch auf die Handelsbeziehungen der Schweiz aus. Umso wichtiger ist für die Schweiz die Möglichkeit zur zwischenstaatlichen Streitbeilegung über den Rechtsweg. Für die Handelsbeziehungen mit der EU, der mit grossem Abstand wichtigsten Handelspartnerin der Schweiz, steht bisher ein solcher Rechtsweg nicht zur Verfügung. Dieser Beitrag untersucht, inwiefern das InstA angesichts der absehbaren globalen Herausforderungen zu einer langfristigen rechtlichen Absicherung der Handelsbeziehungen zwischen der Schweiz und der EU beitragen kann. Aus schweizerischer Perspektive erscheinen zweifellos die rechtliche Absicherung der durch das FHA erreichten Liberalisierung und dessen Modernisierung von grosser Dringlichkeit.

I. Handelsbeziehungen Schweiz - EU im Kontext............................................

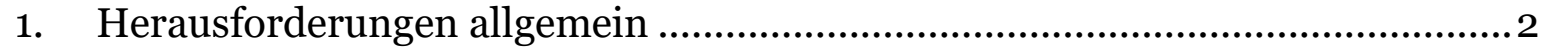

2. Handelspolitische Herausforderungen ....................................................

II. Die WTO, der Handelskrieg und die Schweiz ................................................. 4

1. Die Handelsbeziehungen der Schweiz und die WTO ................................... 4

2. Der Handelskrieg und die Schweiz ..............................................................6 6

3. Auswirkungen des Handelskriegs auf die Beziehung Schweiz - EU ...............8

III. Das InstA und die Handelsbeziehungen Schweiz - EU......................................9

1. InstA: Bedingte Schiedsgerichtsbarkeit in den

Handelsbeziehungen Schweiz - EU...........................................................11

2. InstA: Modernisierung des FHA ............................................................ 12

IV. InstA und die Zukunft europäischer Drittstaaten im EU-Binnenmarkt ............ 15

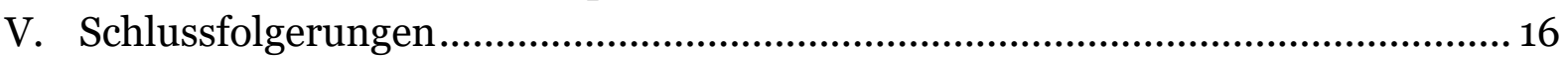

Zitiervorschlag: Charlotte Sieber-Gasser, Handelsbeziehungen Schweiz - EU im globalen Kontext, in: sui-generis 2020, S. 1

URL: $\quad$ sui-generis.ch/116

DOI: $\quad$ https://doi.org/10.21257/sg.116

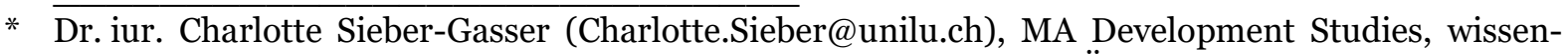
schaftliche Oberassistentin und Lehrbeauftragte im Fachbereich Öffentliches Recht, Universität Luzern. Herzlichen Dank, Simon Guilliard, für die wertvollen Anregungen und die kritische Durchsicht.

Dieses Werk ist lizenziert unter einer Creative Commons Namensnennung - Weitergabe unter gleichen Bedingungen 4.o International Lizenz. 


\section{Handelsbeziehungen Schweiz - EU im Kontext}

1 Über den Entwurf des «Abkommens zur Erleichterung der bilateralen Beziehungen zwischen der Europäischen Union und der Schweizerischen Eidgenossenschaft in den Bereichen des Binnenmarkts, an denen die Schweiz teilnimmt» (InstA) ${ }^{1}$ zwischen der Schweiz und der Europäischen Union (EU) wird seit Dezember 2018 schweizweit intensiv diskutiert. In die politische Diskussion über die Vor- und Nachteile des Vertragsentwurfs fliessen Stellungnahmen der öffentlichen Konsultation ${ }^{2}$ sowie Ergebnisse diverser Rechtsgutachten ${ }^{3}$ und -expertisen 4 mit ein. Im Fokus der Dis-

1 Abkommen zur Erleichterung der Bilateralen Beziehungen zwischen der Europäischen Union und der Schweizerischen Eidgenossenschaft in den Bereichen des Binnenmarkts, an denen die Schweiz teilnimmt (InstA) vom 23. November 2018 (inoffizielle Übersetzung; der Originaltext auf Französisch ist massgebend).

2 EDA, Direktion für europäische Angelegenheiten, Institutionelles Rahmenabkommen: Stellungnahmen, 2019.

3 Siehe Philipp Zurkinden, Institutionelles Abkommen - Rechtsgutachten zuhanden der WAK$N$; Fragen zu Staatsbeihilfen und zum Freihandelsabkommen $\mathrm{CH}-\mathrm{EU}$ sowie zu weiteren Fragen, Prager Dreifuss, 19. Februar 2019; Nicolas Levrat, Avis de droit du Professeur Nicolas Levrat rédigé à la demande de l'Association Suisse des locataires, 8. März 2019; Carl Baudenbacher, Rechtsgutachten zur Streitentscheidungsregelung des InstA zu Handen der Kommission des Nationalrates für Wirtschaft und Abgaben WAK, 6. Februar 2019; Andreas Glaser, Gutachten zur Unterstellung der Genehmigung des Institutionellen Abkommens zwischen der EU und der Schweiz unter das obligatorische Referendum, 3. Mai 2019.

4 Siehe auch Aussenpolitische Kommission des Nationalrates, Öffentliche Anhörungen zum Institutionellen Abkommen zwischen der Schweiz und der Europäischen Union, Medienmitteilung 18. Dezember 2018; Christa Tobler/Jacques Beglinger, Brevier zum institutionellen Abkommen Schweiz - EU, 12. Oktober 2018; Thomas Cottier, Der Rechtsschutz im Rahmenabkommen Schweiz-EU, Die Schweiz in Europa, 8. Januar 2019. kussion steht dabei insbesondere die Bedeutung des Abkommens für die staatlichen Beihilfen, die flankierenden Massnahmen und die Unionsbürgerrichtlinie.

2 Der regulatorische und politische Kontext des Abkommens stand bisher weniger im Fokus der Diskussionen. Dabei deutet vieles darauf hin, dass insbesondere die InstA-Bestimmungen betreffend das Freihandelsabkommen von 1972 mit der EU (FHA) 5 von zu grosser Dringlichkeit sind, als dass ein zuletzt vorgeschlagenes Interimsabkommen - ein sog. «Memorandum of Understanding», welches den status quo der bilateralen Beziehungen einfrieren würde, bis die Verhandlungen über Brexit konkretere Gestalt angenommen haben ${ }^{6}$ - als gangbarer Ausweg erschiene.

\section{Herausforderungen allgemein}

3 Die EU sieht sich gegenwärtig mit einer «Polykrise» konfrontiert, in welcher die verschiedenen Herausforderungen nicht nur gleichzeitig auftreten, sondern sich auch gegenseitig befeuern.7 So droht nach deren zwischenzeitlicher Bewältigung eine neuerliche Verschärfung der globalen Finanz- und Flüchtlingskrisen. ${ }^{8}$ Aber auch die Auswirkungen der Klima-

5 Abkommen zwischen der Schweizerischen Eidgenossenschaft und der Europäischen Wirtschaftsgemeinschaft vom 22. Juli 1972 (FHA; SR 0.632.401).

6 Michael Ambühl/Daniela Scherer, Ein Interimsabkommen könnte den Rahmenvertrag mit der EU retten, Gastkommentar, NZZ, 25. November 2019.

7 Zum von Jean-Claude Juncker geprägten Begriff der Polykrise, siehe auch: Jean-Claude Juncker, Speech by President Jean-Claude Juncker at the Annual General Meeting of the Hellenic Federation of Enterprises (SEV), 21. Juni 2016.

8 Siehe z.B. Stefan Lehne, The EU Remains Unprepared for the Next Migration Crisis, Carnegie Europe, 3. April 2018; Wolfgang Münchau, The Unbreakable, Unsustainable Eurozone, Financial Times, 28. April 2019. 
erwärmung9 und der Digitalisierung10 sind spürbar, hinzu kommen Spannungen im Bereich der globalen Sicherheit. ${ }^{11}$

4 Zusätzlich stellt der Brexit eine immense politische, wirtschaftliche, aber auch regulatorische Herausforderung dar nicht nur für die EU und das Vereinigte Königreich Grossbritannien und Nordirland (nachfolgend: Vereinigtes Königreich), sondern für ganz Europa, die Schweiz inbegriffen. Die EU selbst befindet sich in einer Verfassungskrise, seit sich einzelne Mitgliedstaaten über fundamentale Prinzipien des EU-Rechts hinwegsetzen. ${ }^{12}$ Gleichzeitig ist die EU aber spätestens seit den letzten Parlamentswahlen politisch gespalten, was notwendige Reformen erschwert. ${ }^{13}$

5 Stabile politische und wirtschaftliche Beziehungen zwischen der EU und ihren europäischen Nachbarstaaten sind für die Bewältigung dieser Herausforderungen von grundlegender Bedeutung und liegen somit im vitalen Interesse des gesamten Kontinents Europa. Nichtsdestotrotz gelingt es seit Jahren nicht, die politischen und wirtschaftlichen Beziehungen zwischen der EU und der

Siehe z.B. Stephen Pope, Climate Change Divides Europe, Forbes, 7. Juli 2019.

10 Siehe z.B. Bitten Thorgaard Sørensen, Digitalisation: An Opportunity or A Risk?, Journal of European Competition Law \& Practice, 2018, S. 349-350.

11 Siehe z.B. Hans-Joachim Spanger, Ways out of the Crisis: Recalibrating European Security, PRIF Blog, 23. April 2019.

12 Siehe z.B. von Armin Bogdandy/Michael Ioannidis, Das systemische Defizit. Merkmale, Instrumente und Probleme am Beispiel der Rechtsstaatlichkeit und des neuen Rechtsstaatlichkeitsaufsichtsverfahrens, Zeitschrift für ausländisches öffentliches Recht und Völkerrecht, 2014, S. 283328.

13 Siehe z.B. Sébastien Platon, European Elections, European Values, and Brexit, Blog, DCU Brexit Institute, 5. Juni 2019.
Schweiz auf ein neues rechtliches Fundament zu stellen und damit für Rechtssicherheit und politische Stabilität zu sorgen. Dies soll sich nun mit dem InstA ändern.

\section{Handelspolitische Herausforderungen}

6 Wie nachfolgend erläutert wird, ist das drohende Auseinanderbrechen des regelbasierten globalen Handelssystems für die Einordnung des InstA in den globalen Kontext von besonderer Bedeutung. ${ }^{14}$ In diesem Zusammenhang ist die Schweiz zusammen mit anderen europäischen Drittstaaten direkt davon betroffen, dass die EU die Interessen ihrer Mitgliedstaaten vor diejenigen des Kontinents stellt. Dabei zeigt sich, dass die EU bisher die Option nicht vorsieht, europäische Drittstaaten ohne implizite Voraussetzung einer (zukünftigen) EU- oder EWR-Mitgliedschaft weitreichend in den EU-Rechtsraum zu integrieren. ${ }^{5}$ Sie tut dies mit guten Gründen, denn eine Spaltung Europas in EU-Mitglieder und Nichtmitglieder würde sich langfristig sowohl auf den Wohlstand, wie auch auf das politische Gewicht Europas negativ auswirken. ${ }^{16}$

14 Siehe z.B. Die Volkswirtschaft, Schwerpunkt: Multilateralismus am Wendepunkt, 18. Juli 2019.

15 Benjamin Leruth/Stefan Gänzle/Jarle Trondal, Exploring Differentiated Disintegration in a PostBrexit European Union, Journal of Common Market Studies, 2019, S 1013-1030. Während Assoziationsabkommen der EU teilweise über den Rahmen eines einfachen Freihandelsabkommens hinausgehen, so bleiben sie doch im Umfang deutlich hinter dem EWR/den Bilateralen Abkommen zurück.

16 Siehe auch Christina Eckes, Self-Rule in the 21st Century: How UK Citizens Lose Influence Through Brexit, Blog, DCU Brexit Institute, 13. August 2019. 
7 Stabile Wirtschafts- und Rechtsbeziehungen zwischen der EU und deren europäischen Nachbarstaaten stellen aber gleichzeitig auch ohne die implizite Voraussetzung einer zukünftigen EU- oder EWR-Mitgliedschaft eine Chance dar: Über die Wirtschaftsintegration in den EU-Rechtsraum können die wirtschaftlichen Nachteile der Nichtmitgliedschaft gemindert und kann die gemeinsame europäische Stimme gegen aussen gestärkt werden.

Der im InstA kodifizierte Ansatz zur Teilintegration in den EU-Rechtsraum kommt ohne implizite Voraussetzung einer zukünftigen EU-Mitgliedschaft aus und unterscheidet sich damit deutlich vom Verhandlungskontext der Bilateralen Abkommen I und II. Sofern dieser Ansatz auch langfristig und gegenüber globalen handelspolitischen Herausforderungen Bestand hat, birgt er damit das Potenzial, Europa insgesamt zu stärken, indem er europäischen Drittstaaten eine stabile und zeitlich unbefristete Teilintegration in den EU-Rechtsraum ermöglicht.

\section{Die WTO, der Handelskrieg und die Schweiz}

9 Aus strukturellen Gründen wirken sich sowohl die politischen Entwicklungen innerhalb der WTO wie auch der sog. Handelskrieg zwischen den USA und China auf die Handelsbeziehungen zwischen der Schweiz und der EU aus. Beides trägt dazu bei, dass sich die Dringlichkeit einer Modernisierung des FHA zusätzlich erhöht.

\section{Die Handelsbeziehungen der Schweiz und die WTO}

10 In Ergänzung zum FHA und den Bilateralen Abkommen ${ }^{17}$ mit der EU verfügt die Schweiz über Freihandelsabkommen mit fast allen für die Schweiz wichtigen Export- und Importmärkten ${ }^{18}$ sowie über den minimalen Marktzugang weltweit, welchen die WTO-Abkommen ${ }^{19}$ sicherstellen. Die bi- und plurilateralen Freihandelsabkommen der Schweiz orientieren sich in Struktur und Umfang am multilateralen Rahmen der WTOAbkommen. ${ }^{20}$

11 Die Durchsetzung der in den bi- und plurilateralen Freihandelsabkommen vereinbarten Handelsregeln ist bisher wesentlich vom Streitbeilegungsverfahren der WTO geprägt. Der Streitbeilegungsmechanismus der WTO ist ein zweistufiges Verfahren, welches im Anschluss an den Bericht eines ad-hoc eingesetzten Panels den Parteien die Möglichkeit gibt, den Entscheid an eine zweite Instanz, den Appellate Body, weiterzuziehen. Dessen Entscheidung ist letztinstanzlich

17 Die Bilateralen Abkommen I (Personenfreizügigkeit, Technische Handelshemmnisse, Öffentliches Beschaffungswesen, Landwirtschaft, Forschung, Luftverkehr und Landverkehr) traten 2002 in Kraft. Die Bilateralen Abkommen II (Schengen/Dublin, Automatischer Informationsaustausch AIA, Betrugsbekämpfung, landw. Verarbeitungsprodukte, MEDIA, Umwelt, Statistik, Ruhegehälter und Bildung) traten 2005, 2006 und 2008 in Kraft. Siehe auch Matthias Oesch, Die Europäisierung des schweizerischen Rechts, NCCR Trade Regulation, Working Paper No 2011/05, 2012.

18 Koen Berden/Anirudh Shingal/Charlotte SieberGasser, Verhandlungsposition der Schweiz bei neuen Freihandelsabkommen nur leicht betroffen, Die Volkswirtschaft, 4/2018.

19 Abkommen zur Errichtung der Welthandelsorganisation vom 15. April 1994 (SR 0.632.20).

20 Siehe Charlotte Sieber-Gasser, Democratic Legitimation of Trade Policy Tomorrow, Jusletter, 9. November 2015. 
und für beide Parteien bindend. ${ }^{21}$ Das Fallrecht in Streitfragen betreffend das internationale Handelsrecht ist umfangreich und reicht mittlerweile über siebzig Jahre zurück, ${ }^{22}$ wodurch eine solide Grundlage für die Rechtsdurchsetzung in internationalen Handelsstreitigkeiten entstanden ist. 23

Während sich die Schweiz bei der Anrufung der WTO-Streitbeilegungsinstitutionen bisher sehr zurückhaltend gezeigt hat, 24 nutzen viele der übrigen Mitglieder der WTO das Streitbeilegungsverfahren rege. ${ }^{25}$ Von dieser regen Nutzung der Streitbeilegungsverfahren und dem zugrunde liegenden Konsens, dass die Urteile der WTO-Streitbeilegungsinstanzen grundsätzlich zu respektieren sind, profitiert die Schweiz: Das Verfahren der WTO stellt bisher sicher, dass Handelsstreitigkeiten über den Rechtsweg gelöst werden und nicht über Machtpolitik. ${ }^{26}$

21 Siehe z.B. Werner Schroeder/Pascal Schonard, Die Effektivität des WTO-Streitbeilegungssystems, RIW 9/2001, S. $658 \mathrm{ff}$.

22 Unter dem GATT-Abkommen von 1947 war das Streitschlichtungsverfahren zunächst ein Verfahren in einer Arbeitsgruppe, später dann ein einstufiges ad-hoc Schiedsgerichtsverfahren: WTO, Historic Development of the WTO Dispute Settlement System, 2019. Siehe z.B. auch Andrew Lang, The Judicial Sensibility of the WTO Appellate Body, The European Journal of International Law, 2017, S. $1095 \mathrm{ff}$.

23 Nicolas Diebold/Matthias Oesch, Die Durchsetzung von WTO-Recht durch Schweizer Unternehmen, AJP, S. $1525 \mathrm{ff}$.

24 Matthias Oesch, Handelspolitische Schutzmassnahmen der EU und der Schweiz: Grundlagen, Praxis, Abhängigkeiten, Swiss Review of International and European Law, 2019a, S. $151 \mathrm{ff}$.

25 Seit Inkrafttreten wurden über 551 Streitbeilegungsverfahren eröffnet. Siehe auch Ujal Singh Bhatia, «Unprecedented challenges» confront Appellate Body, chair warns, 22. Juni 2018.

26 Oesch, 2019a (Fn. 21).
13 Allerdings steht der WTO Appellate Body bereits seit längerer Zeit in der Kritik. Beanstandet wird insbesondere, dass das Gericht seine Kompetenzen überschreite, indem es 1) nationales Recht auslege, 2) über die Auslegung der bestehenden Abkommen hinaus neue Verpflichtungen schaffe, und 3) die Tatsachenfeststellungen in den erstinstanzlichen Urteilen ignoriere. ${ }^{27}$ Versuche, das Streitbeilegungsverfahren entsprechend anzupassen, scheiterten bisher am Einstimmigkeitsprinzip der WTO. ${ }^{28}$

14 Der Umstand, dass die USA bereits seit 2011 ihr Veto-Recht bei der Ernennung von Richterinnen und Richtern des Appellate Body einsetzt, verdeutlicht die Dringlichkeit einer Revision des WTOStreitbeilegungsverfahrens. ${ }^{29}$ Spätestens seit dem Amtsantritt von US-Präsident Donald J. Trump 2017 verhindern die USA konsequent die Ernennung von neuen Richterinnen und Richtern. ${ }^{\circ 0}$ Dies hat dazu geführt, dass der Appellate Body ab dem 11. Dezember 2019 nur noch über einen - von eigentlich sieben - amtierenden Richter verfügt. Die zweite Instanz im WTO-Streitbeilegungsverfahren

$27 \overline{\text { Ernst-Ulrich Petersmann, Between «member }}$ driven» WTO governance and "constitutional justice»: Judicial dilemmas in GATT/WTO dispute settlement, EUI Working Papers LAW 2018/04, European University Institute, S. 3-4.

28 Tetyana Payosova/ Gary C. Hufbauer/Jeffrey J. Schott, The Dispute Settlement Crisis in the World Trade Organization: Causes and Cures, 2018, Policy Brief 18-5, Peterson Institute for International Economics PIIE, S. 3. Siehe auch Caroline Glöckle/Aike Würdemann, Die Appellate Body-Krise der WTO - eine Analyse der USKritikpunkte, EuZW, S. 976-982, 2018.

29 Gregory Shaffer/Manfred Elsig, Manfred/Marc A. Pollack, US Threats to the WTO Appellate Body, SSRN, 2017, S. 5.

30 William A. Reinsch/Jack Caporal/Jonas Heering, Article 25: An Effective Way to Avert the WTO Crisis?, Center for Strategic and International Studies CSIS, 24. Januar 2019. 
wurde somit (wenigstens temporär) ab Dezember 2019 aufgehoben. ${ }^{31}$

eil sich aber gleichzeitig an der Rechtsgrundlage für das WTO-Streitbeilegungsverfahren nichts ändert, steht der unterliegenden Partei in einem WTOStreitbeilegungsverfahren weiterhin das Recht zu, den Entscheid der Erstinstanz an den Appellate Body weiterzuziehen. ${ }^{2}$ Da dieser aber nicht mehr urteilen kann, kann die unterliegende Partei damit das Inkrafttreten des erstinstanzlichen Entscheids auf unbestimmte Zeit verhindern. 33

Dies würde sich auch auf die Rechtsmittel auswirken, welche zur Verfügung stehen, um dem WTO-Recht im Streitfall zur Durchsetzung zu verhelfen: Ist ein WTO-Mitglied nicht bereit, die im Streitbeilegungsverfahren festgestellte Verletzung seiner Verpflichtungen im WTORecht innert angemessener Frist zu beseitigen, steht es der obsiegenden Partei zu, Retorsionsmassnahmen zu ergreifen.34 Solche Massnahmen umfassen typischerweise die Aussetzung von Zugeständnissen und anderen Verpflichtungen, sowie Strafzölle. Die vorgesehenen Massnahmen der obsiegenden Partei können wiederum über ein entsprechendes WTO-Verfahren auf ihre Eignung

31 Payosova et al., 2018 (Fn. 28); Peter Van den Bossche, Farewell Speech of Appellate Body Member Peter Van den Bossche, World Trade Organization, 28. Mai 2019.

32 Art. 16 Abs. 4 der Vereinbarung über Regeln und Verfahren für die Streitbeilegung (DSU), Anhang 2 des Abkommens zur Errichtung der Welthandelsorganisation (SR 0.632.20).

33 Nicolas Lamp, Three Scenarios for the Future of the WTO Appellate Body, or: Why WTO Members Should Start Negotiating a DSU 2.O, 2019.

34 Art. 22 DSU. und Verhältnismässigkeit geprüft werden. 35

17 Allerdings steht den Mitgliedern die Möglichkeit zur Ergreifung und Überprüfung von Retorsionsmassnahmen nur offen, wenn zuvor ein Entscheid - entweder des Panels oder des Appellate Bodys - formell angenommen worden ist. 36 Solang der Appellate Body funktionsunfähig ist, steht deshalb der klagenden Partei mangels rechtskräftigem Entscheid kein Rechtsmittel zur Verfügung, um gegen rechtswidrige Massnahmen vorzugehen. Möglich ist deshalb, dass WTO-Mitglieder in einem solchen Fall entweder auf die Streitbeilegungsverfahren in Freihandelsabkommen ausweichen, oder - und angesichts der derzeit vorherrschenden handelskriegsähnlichen Zustände wahrscheinlicher - in Missachtung ihrer DSU-Verpflichtungen dennoch Retorsionsmassnahmen ergreifen. 37

\section{Der Handelskrieg und die Schweiz}

18 Um einen Wechsel hin zu einer zunehmend von Machtpolitik geprägten Rechtsauslegung und -durchsetzung im WTO-Recht zu verhindern, bemühen sich die WTO-Mitglieder um eine temporäre Zwischenlösung. So wird beispielsweise Art. 25 DSU herbeigezogen, welcher die Möglichkeit vorsieht, Handelsstrei-

35 Siehe z.B. Diebold/Oesch, 2008 (Fn. 23); Robert Hennicke, Die Überwachung der Durchführung von Urteilen und Entscheidungen im Völkerrecht am Beispiel der EMRK und des DSU der WTO, MenschenRechtsMagazin, 2016, S. 56 ff. Zu den Möglichkeiten, aber auch Grenzen der Rechtsdurchsetzung über das WTO Streitbeilegungsverfahren und Retorsionsmassnahmen, siehe z.B. Bernhard Zangl, Bringing Courts Back In: Normdurchsetzung im GATT, in der WTO und der EG, Swiss Political Science Review, 2001, S. 49 ff.

36 Art. 22 Abs. 2 DSU.

37 Siehe auch Lamp, 2019 (Fn. 33). 
tigkeiten über ein Schiedsverfahren zu klären. 38 Andere Vorschläge gehen in Richtung eines plurilateralen (also nicht für alle WTO-Mitglieder bindenden) Abkommens über die WTO-Streitbeilegung39, oder in Richtung eines temporären Verzichts auf die Berufungsinstanz. ${ }^{\circ 0}$ Allen vorgebrachten Vorschlägen mangelt es allerdings an einem Mechanismus, welcher dezidiert machtpolitisch agierende Mitglieder in die Schranken zu weisen vermag. Die USA haben sich bisher keiner der verschiedenen Initiativen angeschlossen. ${ }^{41}$

19 Auch die Schweiz beteiligt sich an den Bemühungen um eine Revision des WTO-Streitbeilegungsverfahrens. ${ }^{22}$ Der von der EU und Kanada beschlossenen Interimslösung 43 hat sie sich bisher aber noch nicht angeschlossen. Somit bleibt unklar, welche Rechtsmittel der Schweiz ab Januar 2020 auf multilateraler Ebene offenstehen werden, um ihre Wirtschaft vor diskriminierenden Massnahmen zu schützen.

20 Dies betrifft auch das von der Schweiz initiierte WTO-Streitbeilegungsverfahren

Siehe z.B. Reinsch et al., 2019 (Fn. 30); Lamp, 2019 (Fn. 33); James Bacchus, Saving the WTO's Appeals Process, CATO At Liberty, 12. Oktober 2018.

39 Siehe z.B Jens H. Pohl, Blueprint for a Plurilateral WTO Arbitration Agreement Under Article 25 of the Dispute Settlement Understanding, 2018.

40 Für einen guten Überblick über die verschiedenen Vorschläge siehe Garima Deepak WTO Dispute Settlement - The Road Ahead, New York University Journal of International Law and Politics, 2019, S. $981 \mathrm{ff}$.

41 Deepak, 2019 (Fn. 40).

42 Siehe z.B. Staatssekretariat für Wirtschaft SECO, Positionen der Schweiz in der WTO.

43 Siehe Joint Statement by the European Union and Canada on an Interim Appeal Arbitration Arrangement, Brüssel, 25. Juli 2019. gegen die von den USA seit März 2018 erhobenen zusätzlichen Zölle auf Stahlund Aluminiumprodukte.44 Seit Februar 2019 berät das dafür eingesetzte Panel den Fall.45 Ein für frühestens Mitte 2020 erwartetes erstinstanzliches Urteil könnte rechtlich weiterhin an den voraussichtlich funktionsunfähigen Appellate Body weitergezogen werden.

21 Sowieso ist fraglich, inwiefern Retorsions- oder Schutzmassnahmen überhaupt der Durchsetzung der schweizerischen Wirtschaftsinteressen dienen:

1. Retorsionsmassnahmen stehen rechtlich erst nach einem endgültigen Urteil zur Verfügung. Damit vergeht zwischen Inkrafttreten der rechtswidrigen Massnahmen bis zur Möglichkeit, Gegenmassnahmen zu ergreifen, viel Zeit. Wird die rechtswidrige Massnahme aufgrund der Verurteilung aufgehoben, kann der geschädigte Staat keine Kompensation für den bereits entstandenen Schaden fordern. 46

2. Retorsionsmassnahmen dienen dazu, den durch die rechtswidrige Massnahme entstandenen Schaden über das Aussetzen von Verpflichtungen und Zugeständnissen sowie über Strafzölle zu kompensieren. Dies bedingt, dass mit den genannten Mit-

44 Bundesrat, US-Importzölle auf Stahl und Aluminium: Schweiz leitet WTO-Streitbeilegungsverfahren ein, Medienmitteilung, 10 Juli 2018; United States - Certain Measures on Steel and Aluminium Products, DS556.

45 Siehe auch Matthew Kennedy, Why Are WTO Panels Taking Longer? And What Can Be Done about It?, Journal of World Trade, 2011, S. $221 \mathrm{ff}$.

46 Ausführlicher Diebold/Oesch, 2008 (Fn. 23), S. 1532. 
teln überhaupt eine Kompensation erreicht werden kann. 47

3. Retorsionsmassnahmen dienen auch dazu, zusätzlichen wirtschaftlichen Druck zu schaffen, damit die rechtswidrige Massnahme aufgehoben wird. Grosse Wirtschaftsblöcke liegen in dieser Hinsicht klar im Vorteil, weil sie über das Aussetzen von Verpflichtungen und Zugeständnissen rasch einen substanziellen wirtschaftlichen Druck aufbauen können, während dies für kleinere Volkswirtschaften regelmässig schwierig bis unmöglich ist.

4. Schutzmassnahmen sind temporäre unilaterale und gegenüber allen WTO-Mitgliedern geltende Massnahmen, die dem unmittelbaren Schutz der einheimischen Wirtschaft gegen unfaire oder übermässige Konkurrenz dienen. 48 Schutzmassnahmen bestehen typischerweise aus Kontingenten oder Ausgleichszöllen auf den Import der betroffenen Güter. Ist die einheimische Wirtschaft als Teil einer globalen Wertschöp-

47 So verfügt die Schweiz beispielsweise über einen Handelsüberschuss auf Gütern von 25.37 Mrd. CHF im Jahr 2018 mit den USA, aber über ein Handelsdefizit auf Dienstleistungen von 5.6 Mrd. CHF im Jahr 2018: Die Schweiz importiert 9\% des US-amerikanischen Exports an Computerund Datenverarbeitungs-Dienstleistungen, siehe United States, International Trade Commission, Recent Trends in U.S. Services Trade: 2018 Annual Report, 2018. Weil Retorsionsmassnahmen aber grundsätzlich im betroffenen Sektor ergriffen werden müssen, ist somit unklar, mit welchen konkreten Massnahmen die Schweiz den Schaden der zusätzlichen Zölle auf Stahl- und Aluminiumprodukten der USA kompensieren könnte. Siehe auch Direktion für europäische Angelegenheiten DEA, Schweiz - EU in Zahlen: Statistiken zu Handel, Bevölkerung und Verkehr, Eidg. Departement für auswärtige Angelegenheiten, 2019.

48 Oesch, 2019a (Fn. 21), S. 163 ff.; Diebold/Oesch, 2008 (Fn. 23), S. 1532 ff. fungskette auf den Import von Rohstoffen oder Teilprodukten aus dem Ausland angewiesen, verteuern allfällige Schutzmassnahmen auch die Produktion der einheimischen Wirtschaft und werden damit zum Eigentor. 49

22 Zusammenfassend ist folglich festzuhalten, dass selbst unter der Annahme, dass WTO-rechtliche Massnahmen zur Verfügung stehen, um die schweizerischen Wirtschaftsinteressen im Alleingang durchzusetzen, diese aus Gründen der Exportabhängigkeit entweder nur beschränkte Wirkung entfalten oder der einheimischen Wirtschaft sogar schaden können. Für eine verhältnismässig kleine, nicht in einen Staatenverbund à la EU integrierte Volkswirtschaft wie die Schweiz bleibt somit ein auf festen Regeln beruhendes WTO-System Grundvoraussetzung für die Gewährleistung der Wirtschaftsinteressen. Fehlt der dafür notwendige globale Konsens, gewinnen Rechtsbeziehungen zwischen Gleichgesinnten, z.B. in Freihandelsabkommen, an Bedeutung.

\section{Auswirkungen des Handelskriegs auf die Beziehung Schweiz - EU}

23 Dass die gegenwärtige Krise im regelgebundenen Handelssystem weitreichende Auswirkungen für die Schweiz hat, zeigte

$49 \overline{\text { Unilaterale Kontingente oder Ausgleichszölle als }}$ Schutzmassnahmen gegen die zusätzlichen Zölle auf Stahl- und Aluminiumprodukte der USA würden beispielsweise die Produktion von Stahlund Aluminiumprodukten in der Schweiz verteuern und damit der Wettbewerbsfähigkeit der betroffenen schweizerischen Industrien schaden. Unilaterale Schutzmassnahmen stehen grundsätzlich aus wirtschaftlichen Gründen nur dann sinnvollerweise zur Verfügung, wenn die einheimische Produktion nicht auf den Import aus dem Ausland angewiesen ist. 
sich auch Anfang 2019, als die EU in Reaktion auf die zusätzlichen Zölle auf Stahl- und Aluminiumprodukte der USA unilaterale Schutzmassnahmen in Kraft setzte. Die Schweiz bemühte sich erfolglos darum, von den EU-Schutzmassnahmen ausgenommen zu werden:50 Ausgenommen sind einzig die Mitgliedstaaten des EWR und Entwicklungsländer.51

24 Während die Schutzmassnahmen der EU mutmasslich im Einklang mit WTORecht erlassen worden sind, $5^{2}$ sind sich die EU und die Schweiz uneinig darüber, ob die Massnahmen auch mit Art. 26 i.V.m. Art. 27 FHA vereinbar sind.53 Die Frage wird weiterhin im Gemischten Ausschuss behandelt; weil das FHA bisher kein Streitbeilegungsverfahren vorsieht, kann die Schweiz allerdings kein Rechtsmittel ergreifen, um die Vereinbarkeit der EU-Schutzmassnahmen mit dem FHA zu klären.54 Die Frage bleibt

5o Staatssekretariat für Wirtschaft SECO, $E U$ Massnahmen auf Stahl- und Aluminiumimporten, 2019.

51 Durchführungsverordnung (EU) 2019/159 der Kommission vom 31. Januar 2019 zur Einführung endgültiger Schutzmaßnahmen gegenüber den Einfuhren bestimmter Stahlerzeugnisse.

52 Vereinzelt wird auch innerhalb der WTO infrage gestellt, ob die Voraussetzungen für den Erlass von Schutzmassnahmen erfüllt sind, siehe WTO, Members Review Long List of Safeguard Actions at Committee Meeting, 29. April 2019.

53 Art. 26 FHA besagt: "Bei ernsten Störungen in einem Wirtschaftszweig oder bei Schwierigkeiten, die regional $\mathrm{zu}$ einer schwerwiegenden Verschlechterung der wirtschaftlichen Lage führen können, kann die betroffene Vertragspartei gemäss den in Artikel 27 festgelegten Voraussetzungen und Verfahren geeignete Massnahmen treffen.» Siehe auch SECO (Fn. 50) und Durchführungsverordnung (EU) 2019/159 (Fn. 51), E. 196.

54 Ausführlicher dazu Oesch, 2019a (Fn. 21), S. 168 ff.; Art. 27 FHA sieht die Möglichkeit vor, Schutzmassnahmen zu ergreifen, wenn sich der Gemischte Ausschuss nicht innerhalb von drei Monaten auf ein Vorgehen einigen kann. Aus den obengenannten wirtschaftlichen Gründen aktuell, denn weitere Schutzmassnahmen der EU als Reaktion auf die Handelspolitik der USA sind absehbar. 55

\section{Das InstA und die} Handelsbeziehungen Schweiz - EU

25 Generell ist mit der eingeschränkten oder temporär aufgehobenen - Streitbeilegung in Handelsfragen durch die WTO absehbar, dass sich der Fokus von der multilateralen Ebene auf die bi- und plurilaterale Ebene verschiebt. Damit gewinnen die Streitbeilegungsmechanismen in Freihandelsabkommen an Wichtigkeit. Auch die Schweiz sieht in ihren Freihandelsabkommen Streitbeilegungsverfahren vor. Wie sich zeigt, ist das FHA heute das einzige Handelsabkommen der Schweiz, 56 das für die Streitbeilegung keine Schiedsgerichtsbarkeit vorsieht: bietet diese Rechtsoption der Schweiz allerdings keine Abhilfe.

55 Handelszeitung, Trump macht «Witz» zu 25Prozent-Zöllen auf deutsche Autos, 3. August 2019.

${ }^{6} 6$ Ganz ohne Verweis auf Streitbeilegung: Schweiz - Färöer, Art. 8. 


\begin{tabular}{|c|c|}
\hline Verfahren & Abkommen \\
\hline $\begin{array}{l}\text { Schutzmassnahmen nach } \\
\text { Befassung des Gemisch- } \\
\text { ten Ausschusses }\end{array}$ & Schweiz - EU, Art. 27 \\
\hline Schiedsverfahren & $\begin{array}{l}\text { EFTA - Israel, Art. 25bis1; EFTA - Marokko, Art. 24; EFTA - Palästina, } \\
\text { Art. 29; EFTA - Mazedonien, Art. 31; EFTA, Art. 48; EFTA - Jordanien, } \\
\text { Art. 31; EFTA - Libanon, Art. 34; EFTA - Tunesien, Art. 38; EFTA - SACU, } \\
\text { Art. 37; EFTA - Ägypten, Art. 41; EFTA - Serbien, Art. 33; EFTA - Albanien, } \\
\text { Art. 32; EFTA - Montenegro, Art. } 42\end{array}$ \\
\hline Streitbeilegungsverfahren & $\begin{array}{l}\text { EFTA - Mexiko, VIII Streitbeilegung; EFTA - Singapur, IX Streitbeilegung; } \\
\text { EFTA - Chile, Kapitel X; EFTA - Südkorea, IX. Streitbeilegung; EFTA - Ka- } \\
\text { nada, VIII. Streitbeilegung; EFTA - Kolumbien, 12. Kapitel; Schweiz - Japan, } \\
\text { 14. Kapitel; EFTA - GCC, Chapter 8; EFTA - Ukraine, 9. Kapitel; EFTA - } \\
\text { Peru, 12. Kapitel; EFTA - Hongkong, 10. Kapitel; EFTA - Bosnien und Her- } \\
\text { zegowina, 8. Kapitel; EFTA - Zentralamerikanische Staaten, Chapter 12; } \\
\text { Schweiz - China, Chapter 15; EFTA - Philippinen, Chapter 13; EFTA - Ge- } \\
\text { orgien, Kapitel 12 (noch nicht in Kraft: EFTA - Türkei, Chapter 9; EFTA - } \\
\text { Ecuador, Chapter 11; EFTA - Indonesien, Chapter 11) }\end{array}$ \\
\hline
\end{tabular}

Während die Schweiz mit dem möglichen Wegfall der WTO-Streitbeilegungsverfahren in Bezug auf schätzungsweise 24\% des Handels mit Gütern auf ein Schiedsverfahren in einem Freihandelsabkommen zurückgreifen kann, steht diese Absicherung für die übrigen $60 \%$ des Schweizer Güterhandels mit der EU, wie auch für die $12 \%$ mit den USA nicht zur Verfügung. 57

Illustrativ dafür, was das fehlende Streitbeilegungsverfahren im FHA für die Schweiz bedeuten kann, ist der sog. Steuerstreit: Die EU erachtete 2007 bestimmte kantonale Steuererleichterungen für Holding- und verwandte Gesellschaften in der Schweiz als Verstoss gegen das FHA. Ob sich die EU-Auslegung des FHAs in einem Streitbeilegungsverfahren durchgesetzt hätte, bleibt bis heute

57 Shingal Berden/ Charlotte Sieber-Gasser, 2018, Abb. 2; Direktion für europäische Angelegenheiten DEA, Schweiz-EU in Zahlen: Statistiken zu Handel, Bevölkerung und Verkehr, Juni 2019. höchst fraglich. ${ }^{8}$ Dennoch hatte sich die Schweiz letztendlich aus politischen und wirtschaftlichen Gründen mit der EU darauf geeinigt, die fraglichen Steuererleichterungen abzuschaffen.59 Letztere wurden in einem über mehrere Jahre dauernden Prozess schliesslich mit der vom Volk im Mai 2019 angenommenen STAF-Vorlage abgeschafft. ${ }^{60}$ Ein Streitbeilegungsverfahren hätte der Schweiz 2007 die Möglichkeit eröffnet, sich auf dem Rechtsweg gegen die fragwürdige EU-Auslegung des FHAs zur Wehr zu setzen, womit die rechtliche Seite des sog. Steuerstreits somit innerhalb weniger Monate hätte geklärt werden können.

${ }_{58} \overline{\text { Philipp Roth, Der Steuerstreit zwischen der }}$ Schweiz und der Europäischen Union, Der Treuhänder 2010, S $721 \mathrm{ff}$.

59 Insgesamt dauerte der Steuerstreit über 10 Jahre, siehe z.B. Simon Hirsbrunner, Könnte die Schweiz ein Verbot staatlicher Beihilfen verkraften?, Zeitschrift für Europarecht, 2017, S. 60 ff; Thomas Linder/Cyrill Diefenbacher, Darstellung der EU-Regionalbeihilferegelungen und Schlussfolgerungen für Steuererleichterungen in der Schweiz, Archiv für Schweizerisches Abgaberecht, 2014/15, S. $565 \mathrm{ff}$.

60 Eidgenössisches Finanzdepartement, Steuerreform und AHV-Finanzierung (STAF), 2019. 
1. InstA: Bedingte Schiedsgerichtsbarkeit in den Handelsbeziehungen Schweiz - EU

28

Das InstA kommt damit grundsätzlich zur richtigen Zeit, denn es soll mit einem Streitbeilegungsverfahren zu einer höheren Rechtssicherheit in den bilateralen Wirtschaftsbeziehungen beitragen. Das FHA (und damit der Güterhandel) wurde allerdings auf Wunsch der Schweiz grundsätzlich vom Anwendungsbereich des InstA ausgenommen. ${ }^{61}$ Der Bundesrat ist sich aber der Nachteile des im FHA fehlenden Streitbeilegungsverfahrens bewusst: 62

Für die Interimsphase ab Inkrafttreten des InstA bis zur Inkraftsetzung eines allfälligen modernisierten FHA sieht die Erklärung die Möglichkeit vor, den Streitbeilegungsmechanismus des InstA auch im Bereich des FHA [...] zu nutzen [...]. Voraussetzung ist, dass beide Parteien im konkreten Streitfall damit einverstanden sind. Die Nutzung des InstA-Streitbeilegungsmechanismus unter dem bestehenden FHA wäre grundsätzlich auch im Interesse der Schweiz.

Mit der Ratifikation des InstA bestünde folglich die Option, z.B. ein Streitbeilegungsverfahren über die Vereinbarkeit der EU-Schutzmassnahmen mit dem FHA zu initiieren, unter der Voraussetzung, dass die EU ihr Einverständnis dazu gibt. Ob ein solches Verfahren im Interesse der EU wäre, ist bisher unklar.

$61 \overline{\text { Erläuterungen des Bundesrates zum Institutio- }}$ nellen Abkommen Schweiz-EU, 16. Januar 2019, S. 3 .

62 Ibid., S. 16.
30 Ebenso bleibt fraglich, ob sich die Schweizer Auslegung des FHAs in Bezug auf die EU-Schutzmassnahmen durchsetzen würde. Dass die EU-Schutzmassnahmen grundsätzlich mit dem WTO-Recht vereinbar sein dürften, deutet jedenfalls darauf hin, dass sie grundsätzlich auch mit ihren neueren Freihandelsabkommen vereinbar sind, sofern diese in Bezug auf Schutzmassnahmen weitgehend mit dem WTO-Recht übereinstimmen. ${ }^{63}$

31 Die Besonderheit des FHAs liegt darin, dass das Abkommen 1972 abgeschlossen worden ist, und damit mehr als 20 Jahre vor Inkrafttreten des WTO-Abkommens über Schutzmassnahmen.64 Demnach war es den Vertragsparteien zum Zeitpunkt der Verhandlungen unmöglich, die entsprechenden Bestimmungen des WTO-Rechts in das FHA zu inkorporieren.65 Die Schweiz beruft sich in dieser Frage also auf eine gewissermassen veraltete Bestimmung, welche es in dieser Form heute so in anderen Freihandelsabkommen nicht mehr gibt. Somit ist vorliegend strittig, ob die EU die im FHA für den Erlass von Schutzmassnahmen vorgesehenen Verfahrensbestimmungen

63 Dies ist beispielsweise der Fall in Art. 3.1, EUSingapur; Art. 22, EU-Israel; und in Art. 3.2, EUSüdkorea.

$64 \mathrm{Zu}$ prüfen wäre demnach auch, ob nach den Regeln von Art. 30 der Wiener Vertragsrechtskonvention nicht sowieso die Bestimmungen des WTO-Abkommens über Schutzmassnahmen anwendbar sind. Siehe Wiener Übereinkommen über das Recht der Verträge vom 23. Mai 1969 (SR 0.111).

65 Auch die Schweiz inkorporiert in neueren Abkommen üblicherweise die Bestimmungen über Schutzmassnahmen der WTO-Abkommen, siehe z.B. Art. 18 Ziff. 3, EFTA-Chile; oder Art. 20, Schweiz-Japan. 
eingehalten hat - und nicht, ob Schutzmassnahmen per se zulässig sind. ${ }^{66}$

es sich beim FHA um ein Freihandels- und nicht um ein Marktintegrationsabkommen handelt, erscheint die im InstA vorgesehene Möglichkeit zur Anrufung des Europäischen Gerichtshofs (EuGH) unnötig. Die Zuständigkeit des EuGH entspricht auch nicht dem üblichen Streitbeilegungsverfahren in anderen Freihandelsabkommen der EU.67 Unter diesem Gesichtspunkt ist nachvollziehbar, weshalb der Bundesrat verlangt hat, dass das FHA vom InstA ausgenommen bleibt.

33 Dennoch bleibt offen, weshalb der Bundesrat nicht im Interesse der Rechtssicherheit in die - zumindest vorübergehende - Anwendbarkeit des InstAStreitschlichtungsverfahrens für das FHA eingewilligt hat: Der EuGH wird grundsätzlich vom InstA-Schiedsgericht nur beigezogen in Auslegungsfragen zu Rechtsbegriffen des EU-Rechts. ${ }^{68}$ Da das FHA - anders als etwa das Luftverkehrs-

$66 \overline{\text { Der Bundesrat antwortete auf die Frage, wie die }}$ Ungleichbehandlung der Schweizer Stahlindustrie gegenüber den EWR-Staaten mit den Bilateralen Abkommen vereinbar sei, wie folgt: «Der Warenverkehr zwischen der Schweiz und der EU wird durch das Freihandelsabkommen von 1972 geregelt. Dieses Abkommen verbietet (im Gegensatz zum EWR-Abkommen) die Anwendung von handelspolitischen Schutzmassnahmen nicht und enthält auch keine Sonderbestimmungen über den Handel mit Stahlprodukten. Massnahmen, welche Einfuhren beeinträchtigen, müssen gemäss dem Abkommen allerdings gewisse Voraussetzungen erfüllen (u.a. Schutz überwiegender öffentlicher Interessen, Verhältnismässigkeit).» Antwort des Bundesrats vom 17. August 2016, Interpellation Sauter (IP 16.3508).

67 Siehe auch Christopher Vajda, The EU and Beyond: Dispute Resolution in International Economic Agreements, The European Journal of International Law, 2018, S. 205 ff.

68 Art. 10 Ziff. 3 InstA. abkommen von 1999 -bekanntlich keine Rechtsübernahme von EU-Recht umfasst, ${ }^{69}$ entspräche das InstA-Schiedsgericht in Bezug auf das FHA de facto einem gewöhnlichen Schiedsgericht, wie es die Schweiz auch in ihren anderen Freihandelsabkommen vorgesehen hat.

\section{InstA: Modernisierung des FHA}

34 Geht es nach dem vorliegenden Entwurf des InstA, so werden innerhalb von sechs Monaten nach Unterzeichnung des InstA Verhandlungen über die Modernisierung des FHAs aufgenommen. $7^{\circ}$ Diese Absichtserklärung findet sich auch in der Präambel des InstA, in welcher der gemeinsamen Überzeugung Ausdruck verliehen wird, dass die Modernisierung des FHA erforderlich sei.71 In der das InstA ergänzenden «Gemeinsamen Erklärung EU-Schweiz zu den Handelsabkommen» (Gemeinsame Erklärung) wird die in der Präambel erwähnte Absichtserklärung weiter ausgeführt.

35 So präzisiert Absatz 6 konkret, welche Bereiche die angestrebte Modernisierung des FHAs betrifft:72

69 Mit Ausnahme von Protokoll Nr. 3 zum FHA (Ursprungsprotokoll). Siehe auch Bundesrat, $B e-$ richt des Bundesrates in Beantwortung des Postulats Keller-Sutter [13.4022] «Freihandelsabkommen mit der EU statt bilaterale Abkommen», Juni 2015.

70 InstA, Gemeinsame Erklärung EU-Schweiz zu den Handelsabkommen, Ziff. 11.

71 Instâ, Präambel, para. 4: "In der Überzeugung, dass der kontinuierliche und ausgewogene Ausbau der Wirtschafts- und Handelsbeziehungen zwischen den Parteien auch eine Modernisierung der Instrumente erfordert, die der wirtschaftlichen Beziehung zwischen der Europäischen Union und der Schweiz zugrunde liegen, darunter das 1972 unterzeichnete Freihandelsabkommen (FHA 1972) [...]».

72 InstA, Gemeinsame Erklärung EU-Schweiz zu den Handelsabkommen, Ziff. 6. 
- Marktzugang und Warenverkehr;

- Anwendung von handelspolitischen Massnahmen zwischen den Parteien, insbesondere im Bereich von Schutzmassnahmen;

- Erleichterung des Austauschs und der Zusammenarbeit im Zollwesen, einschliesslich des koordinierten Grenzmanagements;

- Warenursprung: Vereinfachung der Regeln;

- Bestimmungen zur Liberalisierung des Handels mit Dienstleistungen und der Investitionen unter Beibehaltung der einschlägigen allgemeinen Ausnahmen;

- Schutz des geistigen Eigentums;

- Soziale und ökologische Dimension der nachhaltigen Entwicklung, Klimawandel;

- Beschaffungswesen;

- Verbesserung der Mechanismen zur gegenseitigen Anerkennung von Konformitätsbewertungen;

- Staatliche Beihilfen.

Der damit skizzierte Umfang eines modernisierten FHAs entspricht demjenigen von modernen Handelsabkommen der EU, aber auch der Schweiz und der EFTA. Er geht allerdings auch in allen erwähnten Bereichen über den bestehenden Umfang des FHA hinaus. Eine Modernisierung des FHA käme somit - je nach Verhandlungsverlauf - einem Abkommen im Umfang von CETA oder EFTA-Ecuador nahe, was nicht zuletzt auch dem gegenwärtig diskutierten Entwurf für die künftigen Beziehungen zwi- schen der EU und dem Vereinigten Königreich entspricht. 73

37 Neben der Modernisierung des FHAs wird auch die Anwendbarkeit der InstAStreitbeilegung für Handelsstreitigkeiten basierend auf dem bestehenden und dem modernisierten FHA sowohl in der Präambel des InstA, wie auch in der gemeinsamen Erklärung über Handelsabkommen explizit genannt.74 Demnach würden, je nach Umfang der Marktintegration, welche aus einem modernisierten FHA resultiert, die Mehrzahl der Bereiche des modernisierten FHAs vollumfänglich dem InstA unterstellt werden.

38 Weder das InstA noch die Gemeinsame Erklärung oder die Erläuterungen des Bundesrats schaffen Klarheit darüber, welche Teile des modernisierten FHAs dies betreffen dürfte und welcher Streitbeilegungsmechanismus - falls überhaupt - vorgesehen würde für die nicht dem InstA unterstellten Bereiche des modernisierten FHAs.

39 Auf den ersten Blick ist nicht ersichtlich, weshalb der EuGH beigezogen werden müsste in Streitfragen basierend auf einem Abkommen nach dem Vorbild von CETA oder von EFTA-Ecuador. Der entsprechende Hinweis sowohl in der Präambel des InstA, wie auch in der Gemeinsamen Erklärung deutet folglich darauf hin, dass der anvisierte Umfang der Modernisierung wahrscheinlich über den Rahmen von CETA oder EFTA-Ecuador hinausgehen und neben zwischenstaatli-

$73 \overline{\text { Siehe beispielsweise Lisa O'Carroll, Can UK get }}$ "super Canada-plus» trade deal with EU by end of 202O?, The Guardian, 12. November 2019.

74 InstA, Präambel, para. 4; InstA, Gemeinsame Erklärung EU-Schweiz zu den Handelsabkommen, Ziff. 9. 
chen Verpflichtungen auch Rechtsharmonisierung umfassen würde. Andernfalls würde die Bereitschaft der Schweiz, die Zuständigkeit des EuGH in einem modernisierten FHA anzuerkennen, im Widerspruch zur bisherigen Politik stehen.

40 Der Bundesrat bezeichnet die Gemeinsame Erklärung allerdings insgesamt als «rechtlich nicht verbindlich».75 Die Rechtsnatur eines internationalen Akts wird durch den Willen der Parteien bestimmt und geht aus dem Text hervor. ${ }^{76}$ Neben den Erläuterungen des Bundesrats ist deshalb zu beachten, dass Teile der Gemeinsamen Erklärung so formuliert sind, dass sie einen gemeinsamen Willen zur Verbindlichkeit nahelegen und der Präzisierung von einzelnen Bestimmungen im - rechtlich verbindlichen - InstA dienen.77

41 Vergleichbar ist die Gemeinsame Erklärung somit mit der «Political Declaration», welche in den Verhandlungen über den Brexit dem Entwurf des Withdrawal Agreement angehängt ist. Die Political Declaration legt dar, wie die zukünftigen Beziehungen zwischen der EU und dem Vereinigten Königreich ausgestaltet sein dürften. Es ist damit ein per se nur politisch verbindliches Dokument. Da aber

$75 \overline{\text { Erläuterungen des Bundesrats zum Institutionel- }}$ len Abkommen Schweiz-EU, 16. Januar 2019, S. 15 .

76 Exemplarisch dazu Claude Schenker, Praxisleitfaden Völkerrechtliche Verträge, Direktion für Völkerrecht, EDA, Ausgabe 2015, Bern, S. 4.

77 So beispielsweise zur Erforderlichkeit der Modernisierung des FHA in InstA, Präambel, para. 4 i.V.m. InstA, Gemeinsame Erklärung EU-Schweiz zu den Handelsabkommen Ziff. 6; zur Unterstellung des modernisierten FHA unter das InstA in InstA, Präambel, para. 4 i.V.m. InstA, Gemeinsame Erklärung EU-Schweiz zu den Handelsabkommen Ziff. 9.
Art. 184 des Withdrawal Agreement (Stand: November 2019) die Parteien zu Verhandlungen über die künftigen Beziehungen zwischen der EU und dem Vereinigten Königreich nach Treu und Glauben («in good faith») verpflichtet, 78 erhält die zunächst nur politisch verbindliche Political Declaration indirekt auch rechtliche Verbindlichkeit.79

42 Einen identischen Verweis auf Treu und Glauben fehlt im InstA. Hingegen tritt mit Verabschiedung der Gemeinsamen Erklärung diese an die Stelle eines begründeten Antrags über den Ausbau der durch das FHA geschaffenen Beziehungen nach Art. 32 FHA. Demnach würde der Gemischte Ausschuss des FHA beauftragt, den Antrag zu prüfen und Empfehlungen zur Einleitung von Verhandlungen auszuarbeiten. ${ }^{80}$ Während sich der Gemischte Ausschuss folglich immer noch auf ein konkretes Verhandlungsmandat einigen müsste, so würde er sich aber wenigstens von den verbindlich formulierten Teilen der Gemeinsamen Erklärung ( «... les signataires conviennent ...» in Ziff. 9 und Ziff. 10) nicht mehr vollständig lösen können. ${ }^{81}$

43 Über die Präambel im InstA und die Verwendung der Gemeinsamen Erklärung als begründeten Antrag gemäss Art. 32 FHA ist der in der Gemeinsamen Erklärung abgesteckte zeitliche und in-

78 Siehe z.B. House of Commons Library, The Political Declaration on the Framework for the Future EU-UK Relations, 30. November 2018; Art. 184 Withdrawal Agreement, Stand Oktober 2019.

79 Siehe z.B. BrexitCentral, The Political Declaration is not a vague wish list, but an attempt to bind the UK to EU policies, 20. Februar 2019.

80 InstA, Gemeinsame Erklärung EU-Schweiz zu den Handelsabkommen Ziff. 11 i.V.m. Art. 32 FHA.

81 InstA, Gemeinsame Erklärung EU-Schweiz zu den Handelsabkommen Ziff. 9 und Ziff. 10. 
haltliche Rahmen von Verhandlungen über eine Modernisierung des FHA für beide Parteien mindestens politisch verpflichtend. Die Ratifikation des InstA müsste folglich zu Verhandlungen über die Modernisierung des FHA führen; und diese Verhandlungen müssten sich damit befassen, welche Teile des modernisierten FHAs dem Streitschlichtungsverfahren des InstA unterstellt werden. Über die Zeit würde das InstA somit dafür sorgen, dass die Schweiz auch in Bezug auf den Handel mit Waren und Dienstleistungen über einen (unbedingten) Rechtsweg verfügt, um ihre Interessen gegenüber der EU durchzusetzen.

\section{InstA und die Zukunft europäischer Drittstaaten im EU-Binnenmarkt}

44 Parallelen bestehen zwischen den skizzierten zukünftigen Handelsbeziehungen zwischen der EU und dem Vereinigten Königreich bzw. der Schweiz. So sehen sowohl die Gemeinsame Erklärung, wie auch die Political Declaration vor, dass die EU künftig je über ein umfassendes Freihandelsabkommen mit der Schweiz und mit dem Vereinigten Königreich verfügt, welches neben dem klassischen Marktzugang auch eine Teilintegration in den EU-Binnenmarkt inkl. Rechtsharmonisierung umfasst.

In Bezug auf die Rechtsharmonisierung bleibt der EuGH zuständig für die Auslegung von EU-Rechtsbegriffen und es wird grundsätzlich die Übernahme von EU-Beihilferecht und die Abschaffung der flankierenden Massnahmen verlangt in den von der Rechtsharmonisierung erfassten Bereichen. ${ }^{82}$ Basierend auf dem

82 Siehe z.B. Art. 4-5 und Art. 8A-C InstA. gegenwärtigen Entwurf des InstA und dem aktuellen Entwurf des Withdrawl Agreement (bzw. der Political Declaration) würde die Schweiz aber insbesondere wegen dem Abkommen über die Personenfreizügigkeit letztendlich umfassender in den EU-Binnenmarkt integriert sein, als dies für das Vereinigte Königreich der Fall wäre. 83

46 Die Behauptung, dass die Schweiz im InstA weiterhin wie ein Beitrittskandidat behandelt werde, 84 erscheint dennoch nicht zutreffend: Während sich die statische Natur der Mehrzahl der Bilateralen Abkommen I und II mutmasslich damit erklären lässt, dass die Verhandlungsführung damals von einer zeitlich begrenzten Anwendung der Abkommen ausgegangen ist, ${ }^{85}$ wird diese Fehleinschätzung mit dem vorliegenden Entwurf des InstA korrigiert. Das InstA erlaubt eine dynamische Anpassung an das EU-Recht in denjenigen Rechtsbereichen, in welchen sich die Parteien über eine Teilintegration in den EU-Rechtsraum einig werden. ${ }^{86}$ Das langfristige Funktionieren der Rechtsbeziehungen zwischen der EU und der Schweiz ist somit nicht mehr länger

83 Der vielleicht grösste Unterschied dürfte sein, dass das Vereinigte Königreich bisher nicht vorsieht, die Mitgliedschaft in der Personenfreizügigkeit aufrechtzuerhalten, siehe auch Joe Owen, Brexit Deal: Political Declaration on future UKEU Relationship, Institute for Government, 17. Oktober 2019.

84 Zurkinden, 2019 (Fn. 3), para. 24. Vgl. wiederum auch Baudenbacher, NZKart 2019 (Fn. 3), 29.

85 Siehe auch Tobler/Beglinger, 2018 (Fn. 4), S. 7; Matthias Oesch, Die bilateralen Abkommen Schweiz - EU und die Übernahme von EU-Recht, AJP, 2017, S. 638.

86 Art. 4 und 5 InstA; ausführlich dazu Oesch, Matthias, Switzerland - EU Bilateral Agreements, the Incorporation of EU Law and the Continuous Erosion of Democratic Rights, Yearbook of European Law, vol. 38, 2019b, S. $28 \mathrm{ff}$. 
abhängig von einer zukünftigen Mitgliedschaft der Schweiz in der EU.

Der politische Preis für die damit greifbare Rechtssicherheit in den Handelsbeziehungen zwischen der Schweiz und der EU ist allerdings hoch: Aus wirtschaftlichen und - infolge des Handelskriegs und der Schwächung der WTO - aus rechtlichen Gründen ist die Schweiz grundsätzlich auf die Absicherung ihrer Wirtschaftsinteressen gegenüber der EU über den Rechtsweg angewiesen. Wegen ihrer ungleich grösseren Handelsmacht gilt dies aber nicht in gleichem Masse für die Handelsinteressen der EU gegenüber der Schweiz. Die EU kann somit ihre Vorstellungen einer sinnvollen Handelspartnerschaft gegenüber der Schweiz weitgehend durchsetzen, insbesondere solange kein Rechtsweg offensteht, welcher es der Schweiz erlauben würde, gegen Übertretungen der EU vorzugehen. Der Preis für Marktzugang, Rechtssicherheit und Teilintegration ist der teilweise Beitritt zum EU-Rechtsraum ohne entsprechende Mitentscheidungsrechte. 87

Während dies aus Sicht der EU den logischen Weg darstellen mag, europäische Drittstaaten im gegenseitigen wirtschaftlichen Interesse teilweise in den EUBinnenmarkt zu integrieren, bleibt gegenwärtig offen, ob die EU damit im aktuellen politischen Umfeld den Preis nicht zu hoch ansetzt. ${ }^{88}$ Nichtsdestotrotz skizziert das InstA - zusammen mit einem noch zu verhandelnden modernisierten FHA - eine dynamische und langfristige Rechtsbasis für die Handels- beziehungen der EU mit europäischen Drittstaaten.

\section{Schlussfolgerungen}

49 Als globalisierte Volkswirtschaft, welche bisher nicht in eine Staatengemeinschaft integriert ist, ist die Schweiz auf die Durchsetzung ihrer Handelsinteressen über den Rechtsweg angewiesen. Die Politik der USA führte aber Ende 2019 da$\mathrm{zu}$, dass die WTO-Streitbeilegung nicht mehr vollumfänglich funktionsfähig ist, wodurch diese wenigstens temporär mit den in Handelsabkommen vorgesehenen Streitbeilegungsverfahren ersetzt werden muss. Die Schweiz verfügt über keine solchen Streitschlichtungsverfahren in ihren Handelsbeziehungen mit der EU, der mit Abstand wichtigsten Handelspartnerin der Schweiz.

5o Das InstA schafft immerhin mittelfristig und nur in Verbindung mit einem umfassend modernisierten und erweiterten FHA die Grundlage für eine stabile, dynamische und damit langfristige Rechtsgrundlage für die Handelsbeziehungen zwischen der Schweiz und der EU. Der politische Preis für Rechtssicherheit und Marktzugang ist jedoch der teilweise Beitritt zum EU-Rechtsraum ohne entsprechende Mitentscheidungsrechte. Es bleibt gegenwärtig offen, ob die EU damit den Preis nicht zu hoch ansetzt.

87 Siehe auch Oesch, 2019b (Fn. 86).

88 Siehe z.B. auch Anand Menon, An inflexible Brussels is damaging ist own interests over Brexit, Financial Times, 19. November 2019. 